\title{
LA DIFUSIÓN DE LA TEORÍA EVOLUCIONISTA DE LAMARCK EN LA REVISTA LA ABEJA (1862-1870) DE BARCELONA
}

\author{
Agustí Camós Cabeceran \\ Centre d'Estudis d'Història de les Ciènces de la Universitat Autònoma de Barcelona.
}

\section{RESUMEN}

La obra de Lamarck fue difundida, entre los años 1862 y 1865 , por la revista La Abeja dirigida por Antoni Bergnes de las Casas y publicada en Barcelona. En dicha revista aparece explicada la teoría evolucionista de Lamarck tanto en la traducción de su obra, Histoire naturelle des végétaux, como en una serie de artículos anónimos sobre la reproducción de los seres vivos. Estos datos pueden revelar que, en la introducción del evolucionismo en España, la teoría evolucionista de Lamarck tuvo mayor influencia de la que generalmente se ha supuesto.

\section{SUMMARY}

Between 1862 and 1864 Lamarck's works were diffused in Spain through a magazine called $\mathrm{La}$ Abeja which was edited by Antoni Bergnes de las Casas, and published in Barcelona. This may reveal a more important influence of Lamarck's theory on the introduction of evolutionary theories in Spain. La Abeja includes the translation of the Histoire naturelle des végétaux where Lamarck incorporated an early explanation of his theory of evolution; and a series of anonymous articles about the reproduction of living beings, where a more complete explanation about Lamarck's evolutionary theory is contained.

\section{INTRODUCCIÓN.}

La mayor parte de los estudios hechos sobre la introducción de la ideas evolucionistas en España se centran en la introducción del darwinismo'. En estos estudios se produce una cierta identificación entre los términos evolucionismo y darwinismo que conduce a interpretar en clave darwinista ideas evolucionistas de otro origen, especialmente lamarckistas. Distintos autores que han estudiado este tema señalan que es

1 NUÑEZ, D. (1977), El darwinismo en España, Ed. Castalia, Madrid; GLICK, T. (1982), Darwin en España, Ed. Península, Barcelona. 


\section{AGUSTÍ CAMÓS CABECERAN}

a partir de la revolución de 1868 , coincidiendo con la libertad de expresión conseguida en el sexenio revolucionario ${ }^{2}$, cuando se produce la amplia difusión de las ideas evolucionistas en España. También han puesto de manifiesto la presencia de planteamientos evolucionistas algunos años antes, aunque a nuestro parecer no se resalta suficientemente la raíz lamarckista de algunos de ellos.

El presente artículo mostrará la difusión de la teoría de la evolución de Lamarck en la revista barcelonesa La Abeja de Antoni Bergnes de las Casas (1801-1879) en los años 1864 y 1865 . De esta forma comprobaremos cómo otros modelos evolucionistas tuvieron una cierta difusión antes de la amplia penetración de las ideas de Darwin, y pudieron jugar un papel significativo en la introducción de las teorías de la evolución en España.

\section{LA REVISTA $L A A B E J A$}

La Abeja fue la última publicación periódica que impulsó Antoni Bergnes de las Casas (1801-1879). Se publicó regularmente entre 1861 y 1867, aunque fue en 1870 cuando aparecieron los dos últimos ejemplares que concluían el sexto volumen de la revista.

Es sorprendente que ni la Història de la premsa catalana ${ }^{3}$ ni la Historia del periodismo español ${ }^{4}$ recojan la existencia de esta revista, que sin embargo parece que tuvo un importante impacto en la sociedad barcelonesa. Solamente Palau ${ }^{5}$ recoge su existencia, "publicación importante dirigida por el inteligente profesor D. Antonio Bergnes de las Casas". La redacción de la revista era responsabilidad de una llamada "Sociedad Literaria" en la que Bergnes debía desarrollar un papel fundamental, como lo demuestra que la introducción del primer número de la revista, en la que se definen las orientaciones fundamentales, esté firmada por Bergnes 6.

Antoni Bergnes fue un importante personaje de la vida cultural barcelonesa del siglo pasado, profesor de lenguas, catedrático de griego y rector de la Universidad de Barcelona, y reconocido editor. Santiago Olives ${ }^{7}$ hizo un amplio estudio de su vida centrado especialmente en su tarea como helenista y editor. De su biografía cabe resaltar algunos aspectos claves para comprender su contacto con la obra de La-

\footnotetext{
2 NUÑEZ, D. (1977), p. 27. GLICK, T. (1982), p. 13. SALA CATALA, J. (1987), Ideologia y Ciencia Biológica en España entre 1860 y 1881, Madrid, p. 21.

3 TASIS, R.; TORRENT, J. (1966) Història de la premsa catalana. Ed. Selecta, Barcelona.

4 GOMEZ, P. (1967) Historia del periodismo español. Editora Nacional, Madrid.

5 PALAU, A. (1951), Manual del librero hispanoamericano, Llibreria Palau, Barcelona, vol. 1, p. 19.

6 Antoni Rave, sobrino de Bergnes y redactor de la revista, escribe que su tio era el director de $L a$ Abeja en la biografía póstuma de Bergnes que publicó en la revista El Mundo ilustrado en el año 1880.

7 OLIVES, S. (1947), Bergnes de las Casas helenista y editor, CSIC, Barcelona.
} 
marck: su dominio de las lenguas europeas más importantes, su fácil acceso a la cultura francesa, su vinculación con el movimiento protestante cuáquero y su ideología liberal progresista.

En relación con las lenguas, además del catalán y el castellano, dominaba el francés, el inglés, el alemán, el italiano, el griego antiguo y el griego moderno. Este extraordinario conocimiento de lenguas le facilitó el contacto con el mundo cultural europeo coetáneo, con las limitaciones impuestas por los condicionamientos de la situación política española.

El cómodo acceso a la cultura francesa, dado su dominio de esta lengua desde la infancia, fue facilitado además porque buena parte de su familia fueron afrancesa$\operatorname{dos}^{8}$, por la estrecha relación que mantuvo con su primo Ramón Dufour, impresor en una pequeña ciudad del Rosellón, y por los dos viajes que realizó al extranjero entre 1824 y 1830 , en los que estuvo durante algún tiempo en Francia.

En los dos viajes citados permaneció durante bastante tiempo en Londres, donde entró en contacto con los círculos protestantes radicales. Tuvo especial relación con los cuáqueros ${ }^{9}$ con los que mantendría contactos a lo largo de su vida, que influyeron en que parte de sus esfuerzos editoriales se dedicaran a la difusión de la ciencia, a la vez que le facilitaron un acercamiento a las teorías evolucionistas ${ }^{10}$.

Su ideología liberal se puso claramente de manifiesto a lo largo de toda su vida en diversos acontecimientos, algunos de los cuales enumeramos a continuación. Participó en la Milicia Nacional Voluntaria durante el trienio liberal y entre los años 1837 y 1840. De 1833 a 1835 publicó el influyente periódico liberal El Vapor. En 1835, en pleno período revolucionario, fue nombrado concejal del Ayuntamiento de Barcelona. Y durante el sexenio revolucionario fue nombrado Rector de la Universidad de Barcelona por la Junta revolucionaria.

Los otros miembros de la "Sociedad literaria" responsables de la redacción de $L a$ Abeja eran: Antonio Sánchez Comendador (1823-1888), según figura en la revista, catedrático de mineralogía y zoología de la Facultad de Ciencias de la Universidad de Barcelona, pero que desde el año anterior a la aparición del primer número de $L a$ Abeja había sido trasladado a la Facultad de Farmacia; Antoni Rave i Bergnes (?1883), catedrático de física de la Facultad de Ciencias de la misma Universidad, sobrino de Antoni Bergnes; Miquel Guitart i Buch, doctor en medicina, que ya había

\footnotetext{
8 Dos tíos de Bergnes, Bernardo de las Casas y Ramón Dufour fueron miembros de la Junta Extraordinaria de Policía nombrada por el general Duhesne durante la ocupación de Barcelona. Bergnes se casaría más adelante con una hija del primero de ellos.

9 VENTURA, J. (1976), Els heretges catalans, Ed. Selecta, Barcelona, p. 191. VILAR, J. (1994), Intolerancia y libertad en la España contemporánea, Istmo, Madrid, pp. 69-71.

10 CAMOS, A. (1994), La concepció evolucionista de la natura en el programa de difusió de la cultura científica d'Antoni Bergnes de las Casas (1801-1879). Tesis de Master (inédita), dirigida por Jon Arrizabalaga, Seminario de Historia de las Ciencias, Universitad Autónoma de Barcelona.
} 
colaborado con Bergnes en otra publicación periódica, Germania ${ }^{11}$, que también tenía como objetivo la difusión de la cultura romántica alemana, y que puede considerarse como un precedente de La Abeja; Joan Font i Guitart (?-1889), doctor en medicina y ciencias exactas y licenciado en ciencias físico-químicas, admirador de la cultura germánica, que conoció personalmente a Alexander von Humboldt en 1858 durante un viaje que realizó a Alemania y al que dedicó un extenso artículo en la revista ${ }^{12}$.

El objetivo fundamental de la publicación era la difusión de la ciencia y la literatura alemana, como queda claramente reflejado en el epígrafe colocado justamente debajo del título, "Revista científica y literaria ilustrada, principalmente extractada de los buenos autores alemanes".

Estaba dirigida especialmente a profesores y estudiantes de la Universidad ${ }^{13}$, ya que Bergnes, gran conocedor del mundo universitario al que había dedicado buena parte de su vida, era consciente de la necesidad de mejorar la formación del estamento docente, que había sufrido las consecuencias de los difíciles momentos que vivió la Universidad en Cataluña durante la primera parte del siglo XIX, así como el aislamiento que vivió el país durante muchos años a raíz del turbulento curso de los acontecimientos políticos.

Esta publicación, haciendo un gran esfuerzo por dar a conocer la cultura alemana a través de la traducción directa sin recurrir a la traducción francesa, fue muy apreciada y tuvo un gran impacto en el mundo universitario. Jordi Rubió i Balaguer señala en el prólogo de la obra de Olives, la gran deuda intelectual de la generación de su padre, Antonio Rubió i Lluch (1856-1937), con La Abeja ${ }^{14}$. Podemos comprobar la gran importancia que tuvo la divulgación de la ciencia en la revista en la tabla 1 , donde se han clasificado los artículos por materias.

La ciencia y la tecnología por una parte, y la literatura y la poesía por otro, representan dos terceras partes de la revista, y suponen por tanto su núcleo. Frecuentemente se ha sobrevalorado la vertiente literaria de La Abeja en detrimento de su papel como difusor de la cultura científica ${ }^{15}$, que es el aspecto más importante y que ocupa el mayor volumen de artículos en el conjunto de la publicación.

El modelo de difusión científica que pretende seguir la revista es el que desarrolla Alexander von Humboldt en obras como Representaciones de la naturaleza o $\mathrm{El}$ Cosmos, tal y como lo explicita el propio Bergnes en la introducción del primer nú-

\footnotetext{
11 Germania, 1849-1850, Imp. de A. Freixas, Barcelona.

12 FONT i GuITART, J. (1862), Alejandro de Humboldt, La Abeja, 1, pp. 121-138.

13 BERGNES, A. (1862), Introducción, La Abeja, 1, pp. 1-2.

14 Rubio, J. (1947). Prólogo. En OLIVES, S. (1947) p. XXXVIII.

15 En este sentido vease la selección de artículos aparecidos en la revista que refleja OLIVES, $S$. (1947), p. 253, o en la referencia a la revista que incluye PALAU, A. (1951), vol. I, p. 19.
} 
mero. Además en ella se publicaron tres artículos del naturalista alemán ${ }^{16}$ y el artículo de homenaje póstumo de Joan Font i Guitart.

La mayor parte de los artículos de difusión científica, de literatura y de filosofía son traducciones de autores germánicos. Entre los científicos traducidos destacan entre otros Emil Rossmaezler (1806-1867), naturalista alemán y diputado liberal, que en sus memorias recoge que estuvo en Barcelona y conoció a Bergnes; Justus Liebig (1763-1825), químico alemán de quien volvió a publicar Las cartas químicas, que ya había publicado en una obra anterior, Germania; Hermann Burmeister (1807-1892) que se convertiría en uno de los defensores alemanes del evolucionismo deísta. Por otra parte un buen número de los autores alemanes traducidos eran liberales: algunos de ellos intervinieron en la Asamblea Nacional de 1848 y 1849 como miembros del partido demócrata ${ }^{17}$.

En relación con los artículos de historia natural, una buena parte son también de autores alemanes, pero no deja de ser sorprendente la importante presencia de autores franceses en una revista de marcada germanofilia, en la que algunos han visto cierta francofobia ${ }^{18}$. Entre los naturalistas franceses encontramos a Jean Baptiste Lamarck (1749-1824), Charles Nicolas Sonnini (1751-1813) y Charles Mirbel (17761854), autores que habían producido la mayor parte de su obra medio siglo antes. Entre ellos destaca Lamarck, ya que en la revista aparece la traducción de la Histoire Naturelle des Végétaux, una importante obra de este autor. Además, su teoría evolucionista también queda reflejada en otros artículos aparecidos en la revista, tal como veremos más adelante.

Es interesante resaltar que la traducción de la Histoire Naturelle des Végétaux se adelantó casi cincuenta años a la traducción de la obra más conocida de Lamarck, la Philosophie zoologique. Esta obra fue publicada por primera vez en castellano en Valencia por la editorial Sempere en 1911, más de cien años después de la primera edición francesa ${ }^{19}$, en el marco de la recuperación del modelo evolucionista lamarckista de principios del siglo XX. A continuación analizaremos la Histoire Naturelle des Végétaux en el marco de la obra de Lamarck, y su traducción aparecida en La Abeja.

\footnotetext{
16 Humboldt, A. (1863a), "Armonías de la Naturaleza", La Abeja, 2, 1-4 y 73-74. HumBoldT, A. (1863b), "Animales invisibles de la atmósfera y del oceáno", La Abeja, 2, 233-234. HuMBOLDT, A. (1862), "Una Carta de Humboldt", La Abeja, 1, 139-140.

17 JURETSCHEE, H. (1979) "La Abeja, de Bergnes de las Casas o aspectos del germanismo catalán hacia mediados del siglo XIX", Miscel-lania Aramon i Serra, Barcelona, 313-329; pp. 319-320.

18 JURETSCHEE, H. (1979), p. 326.

19 CASINOS, A. (1986), Pròleg. En: LAMARCK, J. (1986), Filosofía zoológica, Alta Fulla, Barcelona, pp. XIV-XVII.
} 


\section{AGUSTÍ CAMÓS CABECERAN}

\section{LA TRADUCCIÓN DE LA HISTOIRE NATURELLE DES VÉGÉTAUX.}

La Histoire Naturelle des Végétaux fue publicada en $1803^{20}$ formando parte de una colección titulada Cours complete d'Histoire Naturelle, que contenía la descripción de los tres reinos de la naturaleza en 80 volúmenes. Entre los naturalistas que colaboraron destacaremos al conde de Buffon (1707-1788), a quien pertenecen los volúmenes correspondientes a la Tierra, el hombre, los cuadrúpedos y los pájaros; a Sonnini, a quien corresponden los reptiles; a Lamarck y a Mirbel, a quienes corresponden los 15 tomos sobre los vegetales con los que se acaba la colección, aunque Lamarck únicamente escribió los dos primeros tomos y Mirbel los trece restantes. Este hecho hemos de relacionarlo con su nombramiento, en 1794, como profesor de insectos y gusanos del Muséum National d'Histoire Naturelle. A partir de esta fecha, nueve años antes de la publicación de la Histoire Naturelle des Végétaux, Lamarck abandonó el estudio de la botánica para dedicarse preferentemente al estudio de los animales no vertebrados. Sin embargo en 1803, en el momento de la publicación de la obra, todavía era reconocido como un eminente botánico, tal como lo pone de manifiesto el propio editor del Cours complete d'Histoire Naturelle, en la advertencia que aparece en el primer tomo dedicado a los vegetales, en el que, además de especificar la autoría de los distintos volúmenes, refiriéndose a Lamarck escribe: “...cet illustre auteur, que la Botanique regrette de voir sortir si souvent de ces domaines...".

Es importante destacar que unos años antes de la publicación de la Histoire Naturelle des Végétaux se produjo un cambio fundamental en el pensamiento de Lamarck, coincidiendo con la profundización de sus estudios de zoología de los invertebrados, la aceptación de la visión transformista de los seres vivos y la formulación de una teoría evolucionista. Este trascendental cambio en la visión de la naturaleza y de los seres vivos ha llamado enormemente la atención a los investigadores ${ }^{21}$, entre otras razones, porque se produjo a una edad sorprendentemente avanzada, poco antes de los 56 años. La primera formulación pública de su teoría evolucionista la hizo en el Discours d'ouverture del curso de zoología del año 1800 en el Muséum National d'Histoire Naturelle, y una formulación completa ya aparece en su obra Recherches sur l'organisation des corps vivants, publicada en 1802. Por esta razón, Lamarck, en la Histoire Naturelle des Végétaux que publica al año siguiente, pone de manifiesto la visión evolucionista, desarrollando la aplicación más amplia que se le conoce de

20 LAMARCK, J. (1803), Histoire Naturelle des Végétaux, Deterville, París.

21 GILLISPIE, CH. (1956), "The formation of Lamarck evolutionary theories", Archives internationales d'Histoire des Sciences, 37, 323-338. MAYR, E. (1972), "Lamarck revisted", Journal of the History of Biology, 5, 55-94. BURKHARDT, R. (1972), "The inspiration in Lamarck's belief in Evolution", Journal of the Histoty of Biology, 5(2), 413-438. BURKHARDT, R. (1977), The spirit of System, Lamarck and the evolutionary Biology, Harvard University Press, pp. 115-142. 


\section{LA DIFUSIÓN DE LA TEORÍA EVOLUCIONISTA DE LAMARCK EN LA REVISTA LA ABEJA}

su teoría de la evolución al reino vegetal. Sin embargo, esto sólo ocurre en el capítulo dedicado a la descripción natural y metódica del reino vegetal, porque tal como señala Burkhardt ${ }^{22}$, la mayor parte de la Histoire Naturelle des Végétaux procede de artículos publicados anteriormente por Lamarck en la Encyclopédie méthodique, entre 1783 y 1792 .

En relación con la teoría evolucionista de Lamarck es necesario señalar que su difusión ha estado condicionada por diversos factores que han dificultado notablemente su comprensión, conduciendo frecuentemente a su caricaturización ${ }^{23}$. Entre estos factores cabe señalar algunos ligados a la propia vida de Lamarck, como su compleja personalidad o su enfrentamiento con Cuvier, pero los más importantes son consecuencia de la consideración de su evolucionismo como perdedor frente a la teoría evolucionista de Darwin, y la mitificación del propio Darwin ${ }^{24}$ y de la llamada revolución darwinista ${ }^{25}$. Sin embargo desde los años setenta diversos autores han realizado importantes estudios sobre la obra de Lamarck en su propio contexto histórico que han permitido conocer mejor su obra y su teoría evolucionista ${ }^{26}$. Entre las ideas fundamentales de su teoría evolucionista destacaremos: la existencia de una fuerza que dirige el proceso de la evolución hacia un aumento de complejidad que se manifiesta en su visión del conjunto de los organismos situados en una serie ${ }^{27}$, la acción del medio que diversifica los organismos, la generación espontánea de los organismos más sencillos y la herencia de los carácteres adquiridos como mecanismo básico de la evolución. Podremos comprobar como todas estas ideas del modelo evolucionista de Lamarck aparecen reflejadas en la revista La Abeja, algunas en la traducción de la Histoire Naturelle des Végétaux como veremos en este apartado, y otras en una serie de artículos anónimos que se analizan en el siguiente apartado.

La traducción de la Histoire Naturelle des Végétaux aparecida en La Abeja de Bergnes ocupa 60 páginas repartidas en 15 capítulos, que aparecieron en los tres primeros volúmenes de la revista, los correspondientes a 1862, 1863 y 1864 . Estos

22 BURKHARDT, R. (1977), p. 249.

23 CAMOS, A. (1993), "El tractament ahistòric de Lamarck en els llibres d'ensenyament secundari", II Trobades d'Història de la Ciència i de la Tècnica, Institut d'Estudis Catalans, Barcelona, 347-351.

24 BARTHElEMY-MAUdAle, M. (1979), Lamarck ou le mythe du précurseur, Editions du Seuil, París, pp 11-15.

25 BOWLER, P. (1992), The non-darwinian revolution, The Johns Hopkins University Press, London, pp. 1-19 y 196-204.

26 MAYR, E. (1972), "Lamarck revisted", Journal of the History of Biology, 5, 55-94. BURKHARDT, R. (1977). BARSANTI, G. (1979), Dalla storia naturelle alla storia della natura; saggio su Lamarck, Feltrinelli, Milan. CORSI, P. (1988), The age of Lamarck. Evolutionary Theories in France 1790-1830, University of California Press (primera edición en Bologna, en 1983).

27 LAURENT, G. (1975), "Lamarck: de la philosophie du continu à la science du discontinu", Revue d'histoire des Sciences, XXVIII, 327-360. 


\section{AGUSTÍ CAMÓS CABECERAN}

capítulos fueron agrupados en tres colecciones: la primera toma el título de la obra, "Historia Natural de los vegetales", la segunda se denomina "El ser y la vida de las plantas", y la tercera "Fisiología vegetal"28. La traducción es bastante fidedigna, aunque habiéndose de publicar en capítulos en una revista hubo de adaptarse a las condiciones de este medio. Así, los títulos de los apartados que se desarrollan en cada capítulo se acumulan al principio del mismo, apareciendo a continuación los diferentes apartados sin ninguna separación, y a menudo fusionando párrafos consecutivos. Esto, que parece obedecer a criterios editoriales, ya que permite al lector de la revista conocer el contenido del artículo leyendo la cabecera, hace muy confusa la lectura. Por otro lado se eliminan algunas referencias a obras del mismo autor y, lo que es más grave, se eliminan algunas páginas de difícil lectura, especialmente del final del segundo tomo de la obra ${ }^{29}$.

Un tipo de error que puede ser bastante significativo para determinar las condiciones en que se produjo la traducción, lo constituyen las confusiones sobre el autor de la obra. En algunos artículos escritos por Lamarck aparece como autor Mirbel, su colaborador ${ }^{30}$; en cambio otros artículos, que sí que son de Mirbel, aparecen bajo el nombre de Lamarck. En otros capítulos, aunque aparece el nombre del verdadero autor, hay un error en relación a la inicial de su nombre apareciendo reiteradamente una "L" en lugar de una "J". La repetición de errores de estas características revela que para el equipo redactor de la revista no se trataba de un autor muy conocido, sugiriendo la posibilidad de que se tratara de una obra traducida algunos años antes, como ocurriera con otras obras que aparecen en La Abeja, como las Cartas químicas de Liebig.

El capítulo que incluye la explicación de la teoría evolucionista, aparece al principio del tercer volumen de la revista. No se trata de una explicación ordenada y completa del modelo evolucionista, si no de la primera vez que aparece escrita en castellano una explicación de la teoría de la evolución de Lamarck hecha por el propio autor. Habremos de esperar cerca de medio siglo para que aparezca una nueva traducción al castellano de una explicación del propio Lamarck, en el marco de la primera traducción de la Philosophie Zoologique.

28 La primera colección consta de dos capítulos publicados en el primer volumen, pp. 293-301 y 333-341; la segunda consta de cinco capítulos, tres en el primer volumen, pp. 378-383, 413-417 y $452-$ 457, y dos en el segundo, pp. 16-17, y 39-42; y la tercera consta de ocho capítulos, siete de ellos publicados en el segundo volumen, pp. 78-81, 123-126, 201-205, 278-282, 361-366, 408-411 y 444-446, y el último en el tercero, pp. 9-12.

29 Cuatro páginas de la introducción (LAMARCK, J. (1803), pp. 1-4), treinta y cinco de una extensa clasificación de hojas (LAMARCK, J. (1803) pp. 31-66), otras cuatro que corresponden a una valoración de la clasificación de Linné (LAMARCK, J. (1803), pp. 247-251), así como las últimas 50 páginas (LAMARCK, J. (1803), pp. 286-335).

30 Esta confusión aparece en cinco capítulos de la segunda colección. 


\section{LA DIFUSIÓN DE LA TEORÍA EVOLUCIONISTA DE LAMARCK EN LA REVISTA LA ABEJA}

En esta explicación Lamarck pone como punto de partida de su concepción evolucionista la visión del conjunto de los organismos situados en una serie de creciente complejidad de organización, producto de la acción de la naturaleza. Es el mismo punto de partida que ya había expuesto en otra obra, Recherches sur l'organisation des corps vivants, y que posteriormente también utilizaría en la Philosophie Zoologique.

\footnotetext{
"Esta gradación real en la organización de los seres vivos debe necesariamente ser considerada como el resultado de la verdadera marcha de la naturaleza" 31 .
}

Expone a continuación la existencia de dos series independientes, una en el reino animal y otra en el vegetal, formadas por lo que llama "las masas" que corresponden a los grandes grupos de clasificación y no por géneros o por especies ${ }^{32}$. Aparece también otra idea clave en su concepción evolucionista, la acción del medio sobre los distintos grupos de organismos como causa de las ramificaciones que aparecen en el camino de la evolución ${ }^{33}$. Diferencia siete clases en el reino vegetal, que va describiendo de una forma ordenada, desde la más simplificada hasta la que él considera la más compleja, destacando cuáles son los nuevos grados de complejidad que adquiere cada clase y cada orden respecto al grupo anterior.

En medio de estas descripciones el autor plantea analogías con el reino animal: no es extraño, si consideramos que, como ya hemos comentado, cuando Lamarck escribe esta parte de la obra, hace ya nueve años que ocupa el puesto de profesor de animales invertebrados en el Museum, y que el desarrollo de su teoría la hizo inicialmente sobre el reino animal:

\footnotetext{
"Así como en la marcha que sigue la naturaleza para efectuar sus producciones va de lo simple a lo compuesto, y de lo menos compuesto a lo más compuesto, es evidente que los animales de corazón unilocular deben preceder a los que tienen un corazón de dos celdillas o dos ventrículos, y éstos ser precedidos por animales que no tienen corazón; así también los vegetales..." 34 .
}

A lo largo de toda la exposición se refiere constantemente a la naturaleza como autora de los organismos, tal como podemos comprobar en frases como: “...el resultado de la verdadera marcha de la naturaleza", o "la marcha que sigue la naturaleza para efectuar sus producciones".

31 LAMARCK, J. (1864), "Historia Natural de los Vegetales". La Abeja, 3, 9-12, p. 9.

32 LAMARCK, J. (1864), p. 9.

33 LAMARCK, J. (1864), p. 9.

34 LAMARCK, J. (1864), pp. 10-11. 


\section{AGUSTÍ CAMÓS CABECERAN}

De esta forma, podemos afirmar que en la revista La Abeja del año 1864 aparecen, en palabras del propio autor, algunas de las ideas fundamentales del modelo evolucionista de Lamarck. Evidentemente no se trata de una exposición completa de su teoría, pero sí de la aplicación más completa que hizo al reino vegetal, a lo largo de su vida.

No conocemos con seguridad el nombre del autor de la traducción, ya que no aparece en ninguno de los capítulos. Podría tratarse tanto de Bergnes, de Guitart o de Sánchez Comendador, ya que nos consta que los tres habían traducido artículos de historia natural en los primeros números de la revista. Sin embargo es necesario resaltar la gran experiencia que tenía Bergnes en esta tarea, pues en su juventud había traducido las obras completas de Buffon ${ }^{35}$ y la Histoire Naturelle du genre humain de Virey ${ }^{36}$. Por otro lado, los errores que aparecen en la traducción, especialmente los relativos al autor de la obra y a su nombre, repetidos capítulo tras capítulo, hace pensar que Bergnes utilizó un material probablemente traducido años antes por él mismo u otro colaborador, para incluirlo en la revista cuando ya no tenía muy presente el nombre de su autor.

\section{El EVOLUCIONISMO LAMARCKISTA EN UNA COLECCIÓN DE ARTÍCULOS SOBRE LA REPRODUCCIÓN DE LOS SERES VIVOS.}

Entre los años 1864 y 1865 aparece bajo el título de "Funciones de generación y reproducción", una colección de nueve capítulos en los que se desarrollan aspectos generales sobre la reproducción de los seres vivos, y que se centran especialmente en la reproducción del hombre. En ellos se exponen sucesivamente ideas generales sobre la reproducción, las diferencias entre los sexos, el instinto de reproducción, la concepción y la fecundación, el desarrollo del feto, sus partes anexas, el embarazo y, por último, el parto ${ }^{37}$. Como en buena parte de los artículos publicados en La Abeja estos aparecen anonimamente.

A lo largo de la colección se van intercalando las ideas claves del modelo evolucionista de Lamarck, de una forma bastante clara y completa. Veámoslo a continuación.

La primera idea clave del modelo evolucionista de Lamarck que aparece en esta colección de artículos, es la generación espontánea de los organismos más sencillos

\footnotetext{
35 BUfFON, (1832), Obras completas de Buffon, Imp. de A. Bergnes, Barcelona, 58 volúmenes

36 VIREY, J. (1842), Historia Natural del género humano, Imp. de A. Bergnes, Barcelona, 2 volúmenes.

37 "FUNCIONES de generación y reproducción de los seres vivos". (1864-1865), La Abeja, 3, 125 126, 213-217, 291-295, 337-342, 400-404, 442-446; La Abeja, 4, 47-49, 123-125, y 187-189.
} 
que aparece explicada de una forma bastante detallada ${ }^{38}$. Es interesante resaltar que en esta parte se incluye una referencia a Antoni Martí Franqués (1750-1832) ${ }^{39}$, el destacado químico y botánico ilustrado tarraconense, al describir diferentes experiencias sobre la generación espontánea. Este hecho nos sugiere que el autor del artículo era un médico del país, o bien que su traductor añadió esta cita, siendo conocedor del prestigio que tenía en Barcelona este importante personaje. Concluyen las explicaciones relacionadas con la generación espontánea, con la defensa que hicieron de este fenómeno Lamarck y Geoffroy Saint-Hilaire ${ }^{40}$.

También se reflejan en el artículo otras ideas claves en el modelo evolucionista de Lamarck, como la existencia de una fuerza que dirige constantemente a los organismos vivos hacia una mayor complejidad, la herencia de los caracteres adquiridos y la existencia de un enorme período de tiempo en la historia de la vida ${ }^{41}$.

En el capítulo quinto aparece otra idea importante, la modificación que sufren los seres vivos por acción del ambiente. Añade además la necesidad de larguísimos períodos de tiempo para poder observar las modificaciones producidas:

\begin{abstract}
"Finalmente, en el día, Lamarck profesa que los vegetales y animales cambian de continuo por las influencias de los climas y de los alimentos, por los efectos de la domesticidad y por el cruzamiento de las razas. Si las especies actuales nos parecen constantes, depende, dice él, de que los climas y las demás circunstancias que modifican aquellas especies no obran en ellas hasta después de un enorme espacio de tiempo; y que de consiguiente se necesitan muchas vidas humanas para presenciar aquellas modificaciones y poderlas dar por bien averiguadas. Según él, los efectos bien examinados de los climas, de los alimentos etc., sobre los vegetales y animales, no permiten negar categóricamente aquellas mutaciones; y lo que en historia natural se llama especies perdidas, puede no sean más que nuestras especies actuales antes de haber sido modificadas. De otra parte esta opinión de Lamarck se halla en armonía con la que ha emitido relativamente al origen de los seres organizados: pues como el movimiento vital, según él, tiene por atributo el ir complicando siempre de más a más la organización, es necesario que las especies vayan también cambiando de continuo."42
\end{abstract}

En este último párrafo aparecen claras referencias a algunas de las disputas que sostuvieron Lamarck y Cuvier. La surgida a raíz del estudio de los cuerpos de los

38 FUNCIONES (1864), p. 341.

39 Martí Franqués, además del famoso estudio sobre la composición del aire, realizó otros importantes estudios entre los que cabe señalar los que hizo sobre la reproducción sexual de los vegetales y sobre la producción artificial de plantas a partir de materia inorgánica, a los que posiblemente se refería el autor del articulo. QuiNTANA, A. (1935), "Antoni de Martí i Franquès (1750-1832) Memòries originals. Estudi biogràfic i documental", Memòries de l'Acadèmia de Ciències $i$ Arts de Barcelona, 24.

40 FUNCIONES (1864), pp. 125-126.

41 FUNCIONES (1864), p. 341.

42 FUNCIONES (1864), p. 400. 


\section{AGUSTÍ CAMÓS CABECERAN}

animales momificados traídos desde Egipto a París por la expedición de Napoleón, y la que sostuvieron sobre la diferente explicación que daban a la evidencia fósil de la existencia de las denominadas especies perdidas ${ }^{43}$.

Es interesante constatar que el autor propone una visión que podría hacer compatible el evolucionismo con un Dios creador, idea que ya había tenido un importante eco en Inglaterra y en Alemania a raíz de la publicación de Vestiges of the Natural History of Creation de Robert Chambers (1802-1871), y que a final de siglo intentarían recuperar muchos neolamarckistas. Podemos comprobarlo cuando el autor, después de referirse a la fuerza que hace que los organismos se vayan transformado en otros cada vez más complejos, añade:

\footnotetext{
"De consiguiente un solo y mismo acto hubiera bastado al Creador para producir la serie tan variada de seres vivos, y aún para aumentarla..." 44
}

Además de la explicación de las ideas evolucionistas de Lamarck, aparece expuesta también la idea de unidad de organización de Geoffroy Saint-Hilaire ${ }^{45}$. Estos planteamientos tuvieron bastante influencia no sólo en Francia y Alemania sino también en determinados sectores sociales de Inglaterra donde llegaron juntamente con los planteamientos evolucionistas de Lamarck ${ }^{46}$.

Como hemos visto, a lo largo de este conjunto de artículos dedicados a temas relacionados con la reproducción, tales como la descripción de los órganos sexuales, la fecundación o el desarrollo del feto, aparecen de forma reiterada explicaciones en torno a los planteamientos evolucionistas de Lamarck. Esta insistencia en tratar un tema alejado del objetivo fundamental de estos artículos, pone de manifiesto que su autor tenía la voluntad de difundir la teoría evolucionista de Lamarck.

El autor de esta colección de artículos debió tener una buena formación científica, siendo presumiblemente un médico, fuertemente influido por las ideas de Lamarck y de Geoffroy Saint-Hilaire, y con un amplio conocimiento de las ideas y experimentos que sobre el tema de la reproducción habían circulado por Europa. Por otro lado, podemos situarlo cronológicamente, ya que aparecen una serie de referencias a hechos que el autor considera muy recientes que nos hacen pensar que fueron escritos algunos decenios antes de su publicación en la revista; así refiriéndose a Lamarck

43 Sobre estas polémicas hay interesantes estudios que aparecen en obras de BURKHARDT, R. (1972) pp. 419-426, y LAURENT, G. (1985), "Cuvier y Lamarck: la querella del catastrofismo", Mundo científco, 66, 136-144.

44 FUNCIONES (1864), p. 341

45 FUNCIONES (1864), p. 129.

46 DeSMOND, A. (1989), The politics of evolution, The University of Chicago Press. 
escribe: "En estos últimos tiempos Lamarck ha tratado de resolver la cuestión"47, o finalmente, "en el día, Lamarck profesa que..."48. Lamarck murió en 1829, y la última obra en la que expuso su teoría evolucionista fue el primer volumen de la Histoire naturelle des animaux sans vertèbres, publicada en 1815. Asimismo, al citar una serie de experimentos sobre la fecundación de huevos de rana realizados antes de 1824 por Jean Baptiste Dumas (1800-1884) y Jean Louis Prevost (1790-1850), se refiere a "una serie de experimentos nuevos" 49 , y al referirse a determinadas ideas sobre la fecundación defendidas por el Dr. Millot, que murió en 1811, escribe: "que han sido de nuevo preconizadas en nuestros días por el Dr. Millot"50.

Este conjunto de datos nos permite pensar que el autor de esta colección de artículos podría encontrase entre los médicos radicales ingleses de la primera mitad del siglo XIX, o estar claramente influenciado por ellos, ya que como ha estudiado Desmond ${ }^{51}$, entre este colectivo estuvo muy presente la obra de Lamarck y la de Geoffoy Saint-Hilaire. Bergnes pudo haber entrado en contacto con estos sectores en sus estancias en Inglaterra.

Otro personaje que pudo participar en la traducción o autoría de estos artículos fue Pere Felip Monlau (1808-1871), el conocido higienista, que fue uno de los colaboradores más importantes de Bergnes ${ }^{52}$. Este médico tuvo que exiliarse en varias ocasiones, y entre 1838 y 1839 residió en París donde pudo ponerse en contacto con círculos neobuffonianos franceses en los que la obra de Lamarck todavía estaba presente, y viajó a Londres donde pudo contactar con médicos radicales y sectores de la clase obrera inglesa entre los que las teorías de Lamarck también eran conocidas.

Además, en la época en que suponemos que se pudo escribir la colección de artículos, Monlau había traducido al castellano un libro de obstetricia de A. Velpau ${ }^{53}$, en el que se incluyen todos los temas que se tratan en los artículos. Monlau también conocía la obra de Martí Franqués citada en el artículo, dada la gran actividad que desarrolló en el mundo cultural barcelonés entre 1820 y 1840, siendo además, desde muy joven, miembro de la Real Academia de Ciencias Naturales y Artes. Es posible que llegara a conocerlo en las tertulias que organizó Martí Franqués durante los años 1829 y 1830 cuando las academias estaban cerradas.

\footnotetext{
47 FUNCIONES (1864), p. 341.

48 FUNCIONES (1864), p. 400.

49 FUNCIONES (1864), p. 294.

50 FUNCIONES (1864), p. 404.

51 DESMOND, A. (1989).

52 OLIVES, S. (1947), p. 116.

53 VELPEAU, A. (1833), Elementos de obstetricia redactados conforme a los principios de tocología y embriología, Imp. JJ. Verdaguer, Barcelona, traducción de P. F. Monlau.
} 


\section{AGUSTÍ CAMÓS CABECERAN}

Monlau también pudo participar en la traducción de la Histoire Naturelle des Végétaux, ya que en los años en que posiblemente se tradujo la obra ya había traducido el manual de botánica de Achile Richard (1794-1852) ${ }^{54}$, médico y botánico francés que fue el sustituto de Mirbel, el botánico que colaboró con Lamarck en la obra traducida. La traducción de Monlau incluye un calendario de floración del propio Lamarck ${ }^{55}$, que nos pone de manifiesto que Monlau ya tenía alguna noticia de la obra del naturalista francés.

\section{BERGNES Y LA TEORÍA DE LA EVOLUCIÓN.}

Aunque no conocemos con certeza ni el nombre del traductor de la Histoire $\mathrm{Na}$ turelle des Végétaux, ni los del autor y el traductor de la colección de artículos sobre la reproducción, es evidente que Bergnes, como director de la revista, era responsable de la publicación de estos artículos en los que se difunden reiteradamente las ideas evolucionistas de Lamarck. Cabe preguntarse entonces, cómo tuvo Bergnes posibilidad de conocer estas teorías.

Hemos de considerar, en primer lugar, que en los años en los que se estaban publicando todos estos artículos en La Abeja, se estaba produciendo, en buena parte de Europa, el intenso debate sobre el evolucionismo provocado por la aparición en 1859 de The origin of Species de Darwin. Este debate no aparece reflejado de ninguna forma en la revista, ya que no encontramos ninguna referencia a la teoría evolucionista de Darwin. Esto nos permite afirmar que, en la época en que se publicaba la revista, Bergnes y sus colaboradores no estaban en contacto con la amplia controversia intelectual que, sobre el evolucionismo, se estaba produciendo especialmente en Inglaterra y en Alemania. Y por tanto, que las ideas evolucionistas procedentes de Europa que aparecen en la revista, han de ser el fruto de contactos anteriores con el mundo intelectual europeo.

Los contactos de Bergnes con la obra de Lamarck pudieron establecerse a partir del contacto directo con la cultura francesa, y también a través de círculos londinenenses que tenían en alta consideración las teorías de Lamarck y de Geoffoy SaintHilaire. Además, sus estrechas relaciones con el movimiento cuáquero le facilitaron la asunción de los planteamientos evolucionistas. Analizaremos por separado cada uno de estos tres aspectos.

Como hemos visto al principio del artículo, Bergnes tenía muchas facilidades para acceder a la cultura francesa. Estas facilidades nos pueden explicar que una de las

\footnotetext{
54 RichARD, A. (1831), Elementos de botànica, Imprenta José Rubió, Barcelona, traducción de P. F. Monlau.

55 RiCHARD, A. (1831), pp. 206-208, 2a. parte.
} 
primeras obras editadas y traducidas por Bergnes fuese la edición en fascículos de las obras completas de Buffon entre 1832 y 1834. Su publicación constituye uno de los mayores esfuerzos editoriales que realizó a lo largo de su vida.

Para la edición de esta obra, Bergnes hubo de entrar en contacto con los círculos neobuffonianos parisinos que continuaban reeditando las obras del conde naturalista francés. En estos círculos, en los que se refugiaban naturalistas marginados de las instituciones científicas francesas por Cuvier, es donde podemos encontrar a diferentes naturalistas que defendieron y divulgaron, al menos parcialmente, planteamientos evolucionistas y en especial la teoría evolucionista de Lamarck ${ }^{56}$. Fue posiblemente a través de estos círculos como Bergnes tuvo conocimiento de la obra de Lamarck.

Un miembro destacado de estos círculos neobuffonianos y defensor de Lamarck, fue Virey, de quien Bergnes tradujo y editó en 1835 la Histoire naturelle du genre humain, que contiene algunos planteamientos evolucionistas. Unos años más tarde, en 1845, el editor barcelonés publicó la traducción de una obra de otro miembro de estos círculos, Bory de Saint-Vincent ${ }^{57}$, que fue uno de los mayores propagadores de la ideas evolucionistas de Lamarck.

Como hemos dicho, el otro camino que le permitió conocer y valorar las ideas evolucionistas fueron sus contactos londinenses. En los viajes de Bergnes a Londres en los años 1824-1825 y en 1830, que constituyen un hecho de gran trascendencia en su vida, entró en contacto con dos personajes que le influenciaron notablemente, Henry Brougham (1778-1868) y William Allen (1793-1864). Ambos pudieron tener una enorme importancia en la aceptación de las ideas evolucionistas y en la valoración de la obra de Lamarck.

Brougham fue un político progresista de origen escocés, que desarrolló una intensa tarea en la Inglaterra del siglo XIX, siendo un claro exponente de la nueva burguesía liberal. Su preocupación por la educación, puesta de manifiesto por la fundación de la Society for the Difusion of Useful Knowledge $e^{58}$, se plasmó igualmente en los esfuerzos por la fundación de la Universidad de Londres. Esta Universidad habría de posibilitar la formación de unos nuevos sectores sociales acomodados que estaban apareciendo en la sociedad industrial inglesa, y que tenían un difícil acceso a Oxford y Cambridge, universidades que por otra parte eran muy refractarias a la incorporación de nuevos conocimientos científicos.

\footnotetext{
56 CORSI, P. (1988), pp. 17-23.

57 BORY de SAINT-VINCENT, J. (1842), Historia de las Islas del Océano, Imprenta del liberal Barcelonés, Barcelona.

58 Bergnes utilizaría algunos materiales de esta sociedad para editarlos en España en la Biblioteca de conocimientos humanos.
} 


\section{AGUSTÍ CAMÓS CABECERAN}

En la nueva Universidad se formaron buena parte de los médicos radicales, un sector de los profesionales de la medicina enfrentado a los médicos aristocráticos que controlaban la profesión, y que luchaban para liberalizar la práctica de la medicina. Entre los médicos radicales de las escuelas privadas de medicina y de la Universidad de Londres tenían una gran influencia la anatomía comparada de Geoffroy SaintHilaire y la zoología de Lamarck ${ }^{59}$. Brougham mantuvo contactos con estos círculos y especialmente con los profesores de la Universidad de Londres, puesto que era un importante miembro del consejo rector de la Universidad, y jugó un destacado papel en la contratación de Robert Grant (1793-1874) como profesor de anatomía comparada $^{60}$. Hemos de recordar que Grant fue el más importante exponente del pensamiento lamarckista en Inglaterra durante la primera mitad del siglo. A través de todo ello Brougham debió conocer y valorar la obra de Lamarck, y dada su notable influencia sobre Bergnes ${ }^{61}$, es posible que el editor barcelonés pudiera entrar en contacto con el pensamiento lamarckista a través de él.

Bergnes, en su vejez, pondría de manifiesto que otro personaje que conoció en su estancia en Londres y que le causó una profunda impresión fue Allen ${ }^{62}$. Este notable químico inglés colaboró con sectores radicales de la clase obrera inglesa, especialmente con Robert Owen y sus seguidores socialistas, entre los que el pensamiento evolucionista de Lamarck también tenía una notable influencia ${ }^{63}$. Allen era además un destacado miembro del movimiento cuáquero con el que Bergnes se encontraba vinculado, y que pudo jugar un papel importante en el acercamiento de Bergnes a las ideas evolucionistas.

El análisis de la doctrina religiosa de los cuáqueros pone de manifiesto que las ideas evolucionistas les eran mucho más fáciles de integrar que a los anglicanos o a los católicos de esta época. Para los cuáqueros lo fundamental era la experiencia religiosa individual, a través de la cual Dios se dirigía al corazón de los hombres ${ }^{64}$, rechazando la autoridad de la Iglesia y de sus ministros, y utilizando la Biblia de forma muy diferente a la interpretación literal que hacían otras confesiones cristianas. Conocían la Biblia y la usaban, pero no como una regla fundamental de fe y conducta, lo cual les daba una relativa libertad de interpretación de la Sagradas Escrituras $^{65}$. Esto les permitía asumir con mayor facilidad una nueva interpretación del

59 DESMOND, A. (1989), p. 31

60 DESMOND, A. (1989), p. 81.

61 OLIVES, S. (1947), p. 23. Por otra parte, el discurso que aparece en una de las primeras obras editadas por Bergnes, Biblioteca de conocimientos humanos, es de Brougham.

62 OLIVES, S. (1947), p. 22.

63 DESMOND, A. (1987), "Artisan Resistance and Evolution in Britain 1819-1848", Osiris 2a. serie, $3,77-110$

64 POUPPARD, P. (ed), (1987), Diccionario de las religiones, Herder, Barcelona, p. 372.

65 New catholic Enciclopedia (1967), The Catholic University of America, vol. VI, p. 201. 


\section{LA DIFUSIÓN DE LA TEORÍA EVOLUCIONISTA DE LAMARCK EN LA REVISTA LA ABEJA}

origen de los distintos seres vivos, y posibilitaba que para muchos de ellos la evolución fuera la mejor manera de comprender la forma en que Dios operó66. De hecho, entre los médicos radicales que defendían las teorías de Lamarck y Geoffroy SaintHilaire y a los que nos hemos referido anteriormente, había algunos cuáqueros como John Epps (1805-1869) y William Lawrence (1783-1867) ${ }^{67}$.

Así pues, Bergnes, gracias a sus tempranos y fáciles contactos con la cultura francesa y a su conocimiento de la historia natural desarrollada en este país, es posible que tuviera conocimiento de la obra de Lamarck desde su juventud, pero, debió ser a través de su relación con Brougham y Allen, y de su vinculación con el movimiento cuáquero, como pudo dar mayor importancia a la teoría de la evolución de Lamarck.

\section{LAMARCK Y LA INTRODUCCIÓN DEL EVOLUCIONISMO EN ESPAÑA.}

Esta presencia del modelo evolucionista de Lamarck en los años sesenta del siglo pasado, que hemos podido comprobar en el caso de la revista La Abeja, pero que pensamos que pudo ser más amplia, nos hace pensar que alguna influencia hubo de tener en la posterior consolidación de la teoría evolucionista en España, de forma análoga a lo que, salvando las distancias, plantea Bowler con respecto a Inglaterra ${ }^{68}$.

Estos datos nos plantean la necesidad de revisar la imagen construida por la historiografía del proceso de introducción de las ideas evolucionistas en Cataluña y en el conjunto de España. En este sentido, los estudios más importantes sobre este tema, de Núñez, Glick y Sala Catalá, se han centrado fundamentalmente en la introducción del evolucionismo darwinista. En estas obras, aunque aparece citado Lamarck, no se resalta suficientemente el componente lamarckista que aparece en buena parte de las referencias que se citan.

Podemos comprobarlo en el primer documento que recoge Núñez en El darwinismo en España, el discurso de José Planellas Giralt, catedrático de historia natural de la Facultad de Ciencias de la Universidad de Santiago de Compostela, en la inauguración del curso académico de $1859^{69}$. En él, no se centra el ataque contra la evolución y el origen animal del hombre en aspectos del modelo darwinista, sino en aspectos del modelo evolucionista de Lamarck como la generación espontánea, y en algunos de los hechos que envolvieron la polémica sobre este modelo a principios del siglo XIX, como los datos obtenidos a partir de la momias egipcias traídas por la expedición de Napoleón al país norteafricano.

\footnotetext{
66 DeSMOND, A; MOORE, J. (1991), Darwin, New York, p. 322.

67 DESMOND, A. (1989), pp. 167-169.

68 BOWLER, P. (1992), The non-darwinian revolution, The John Hopkins University Press, London.

69 NÚÑEZ, D. (1977), pp. 87-90.
} 


\section{AGUSTÍ CAMÓS CABECERAN}

Esta mayor presencia de la teoría de la evolución de Lamarck, también explica la influencia lamarckista que Glick detecta en el artículo que considera la primera alusión a la evolución aparecida en una revista española, la sátira sobre la escala de las transformaciones que se publicó en el Museo Universal 70 en el año 1863.

De igual manera, la conferencia de Letamendi en el Ateneo de Barcelona pronunciada en 1867, que recogen tanto Núñez como Glick ${ }^{71}$, en donde se condenaron las ideas evolucionistas de Lamarck y de Darwin, tenía que responder a una presencia pública de estas teorías en Barcelona, a lo cual La Abeja no había de ser ajeno.

Este componente lamarckista que tienen algunos de los primeros datos que Núñez y Glick ofrecen sobre la introducción de las teorías evolucionistas, y que en general ignoran, lo podemos seguir encontrando en muchos de los datos posteriores que estos autores aportan. Queda por comprobar si estos nuevos aspectos en relación, tanto a la presencia de la obra de Lamarck como a la de su teoría evolucionista, fueron hechos aislados, o bien nos permiten detectar un eco social de estas ideas, mayor del que se ha supuesto hasta ahora en Cataluña y en España.

\section{TABLA 1}

\begin{tabular}{l|rrrrrr|r|r}
\multicolumn{1}{c|}{} & \multicolumn{1}{c}{ I } & II & III & IV & \multicolumn{1}{c}{ V } & VI & TOT. & $\%$ \\
\hline MATERIAS & 79 & 46 & 46 & 47 & 44 & 33 & 295 & 37 \\
Ciencias, tecnolog. & 50 & 41 & 31 & 30 & 56 & 27 & 235 & 30 \\
Oiteratura, poesía & 27 & 69 & 60 & 24 & 32 & 48 & 260 & 33 \\
\hline \multicolumn{1}{c|}{ TotaL } & 156 & 156 & 137 & 101 & 132 & 108 & 790 & 100
\end{tabular}

Tabla de los artículos clasificados por materias aparecidos en cada uno de los seis volúmenes de la revista $L a A b e j a$

70 GLICK, T. (1982), p. 14.

71 NÚÑEZ, D. (1977), p. 26; GLICK, T. (1982), p. 14. 